\title{
Perovskite Core-Shell Nanowire Transistors: Interfacial Transfer Doping and Surface Passivation
}

You Meng, ${ }^{1}$ Zhengxun LAI, ${ }^{1}$ Fangzhou Li ${ }^{1}$ Wei Wang, ${ }^{1}$ SenPo Yip,${ }^{1,2,3,4}$ Quan Quan, ${ }^{1}$ Xiuming Bu, ${ }^{1,4}$ Fei Wang, ${ }^{1,2}$ Yan Bao, ${ }^{5}$ Takuro Hosomi, ${ }^{6}$ Tsunaki Takahashi, ${ }^{6}$ Kazuki Nagashima, ${ }^{6}$ Takeshi Yanagida, ${ }^{6,7}$ Jian Lu, ${ }^{4,5}$ Johnny C. Ho ${ }^{* 1,2,3,4,6}$

${ }^{1}$ Department of Materials Science and Engineering, ${ }^{2}$ State Key Laboratory of Terahertz and Millimeter Waves, and ${ }^{3}$ Centre for Functional Photonics, City University of Hong Kong, Kowloon 999077, Hong Kong SAR

${ }^{4}$ Shenzhen Research Institute, City University of Hong Kong, Shenzhen 518057, P. R. China

${ }^{5}$ Department of Mechanical Engineering, City University of Hong Kong, Kowloon 999077, Hong Kong SAR

${ }^{6}$ Institute for Materials Chemistry and Engineering, Kyushu University, Fukuoka 816-8580, Japan

${ }^{7}$ Department of Applied Chemistry, School of Engineering, University of Tokyo, Tokyo 113-8654, Japan

*Corresponding author: Johnny C.Ho (johnnyho@cityu.edu.hk) 

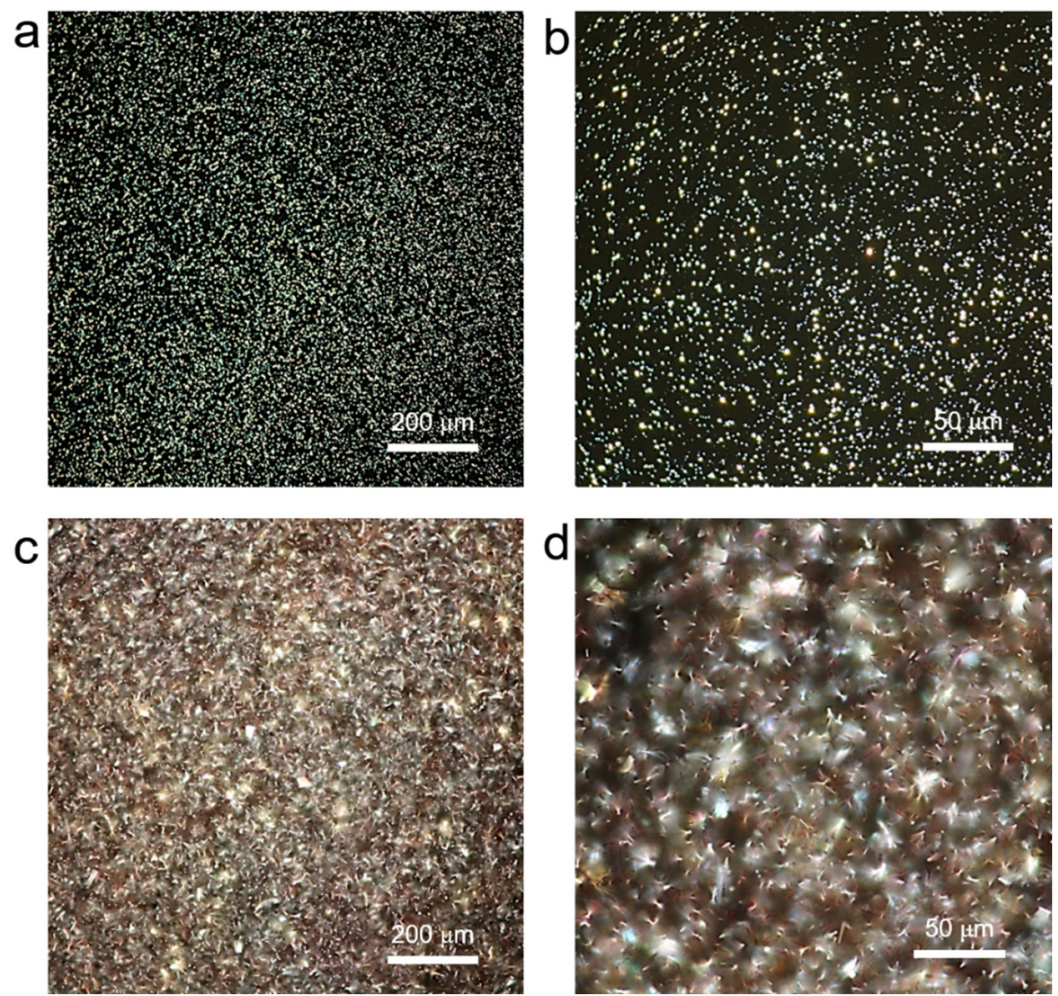

Figure S1. Optical images of $(\mathrm{a}, \mathrm{b}) \mathrm{Sn}$ seeds and (c, d) Sn-catalyzed VLS-growth of vertical CsPbBr3 NWs on $\mathrm{SiO}_{2} / \mathrm{Si}$ substrates (50 nm thick thermally grown oxide).
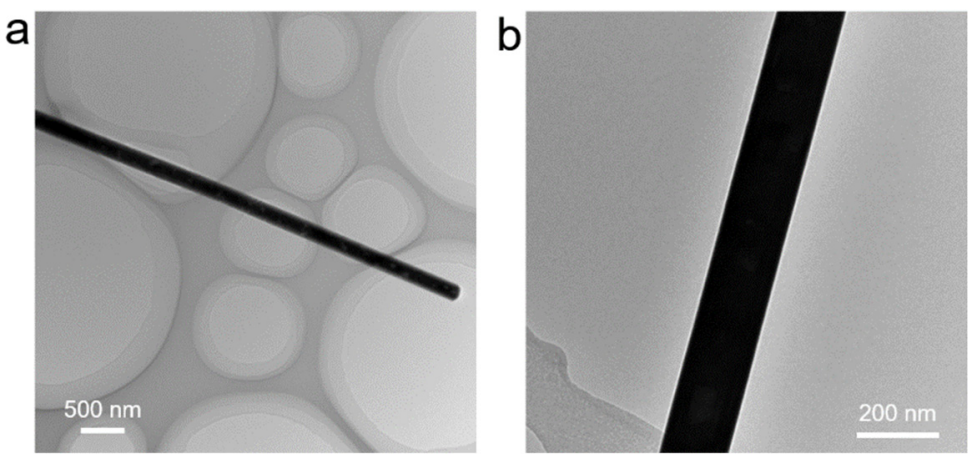

Figure S2. (a, b) TEM images of typical VLS-grown $\mathrm{CsPbBr}_{3} \mathrm{NW}$. 


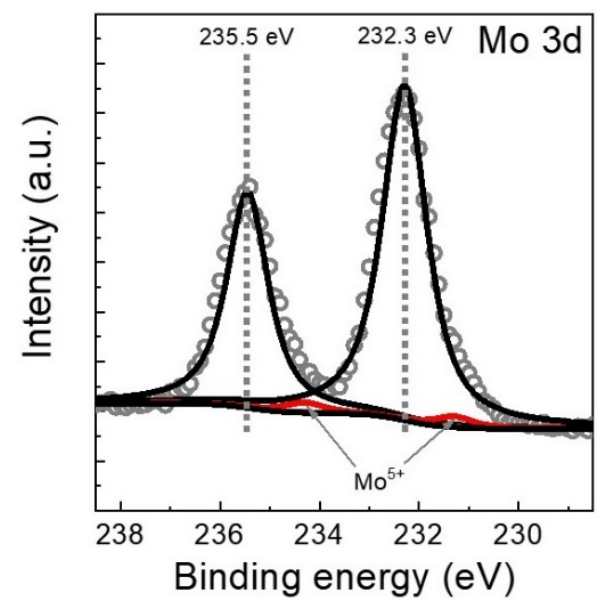

Figure S3. XPS Mo 3d spectrum of 50-nm-thick $\mathrm{MoO}_{3}$ layer deposited on the $\mathrm{SiO}_{2} / \mathrm{Si}$ substrate.
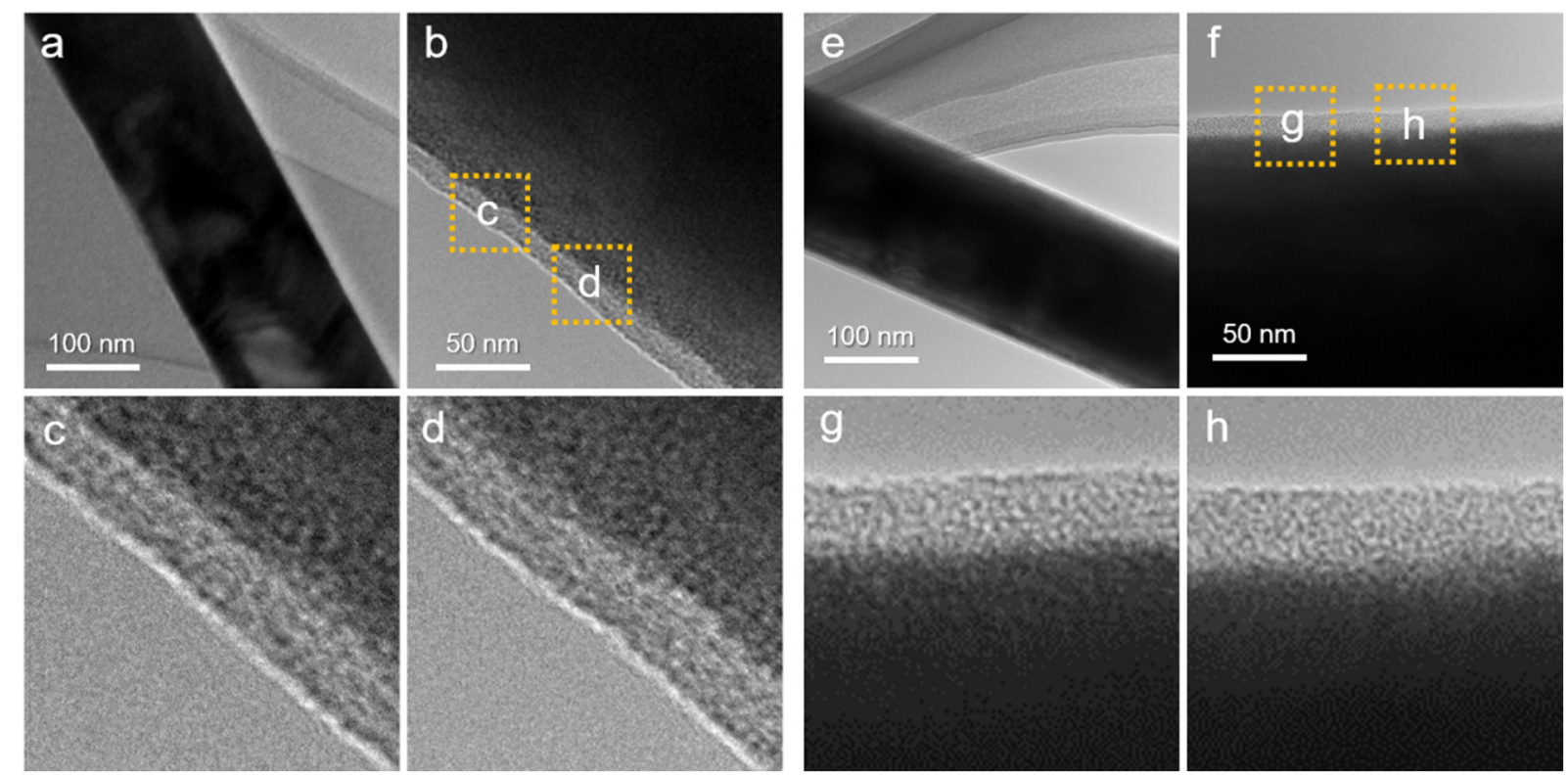

Figure S4. HRTEM images of different regions of the typical $\mathrm{Cs} \mathrm{PbBr}_{3} / \mathrm{MoO}_{3}$ core-shell $\mathrm{NW}$, consisting of a crystalline $\mathrm{CsPbBr}_{3}$ core and a uniform amorphous $\mathrm{MoO}_{3}$ shell.

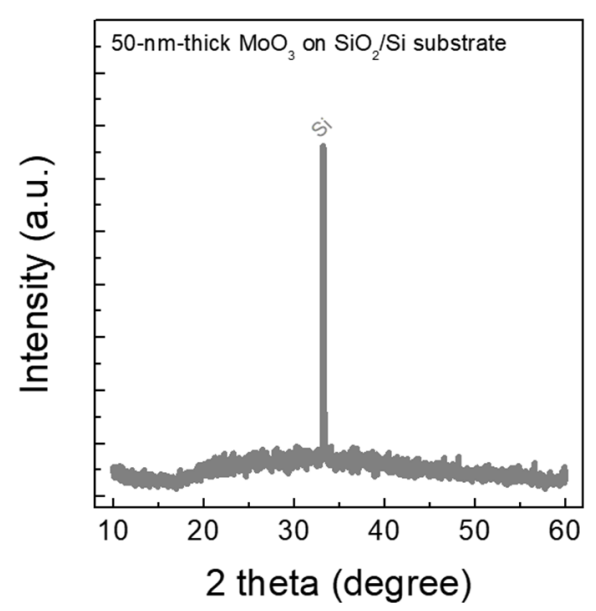

Figure S5. XRD pattern of 50-nm-thick $\mathrm{MoO}_{3}$ layer deposited on the $\mathrm{SiO}_{2} / \mathrm{Si}$ substrate. 


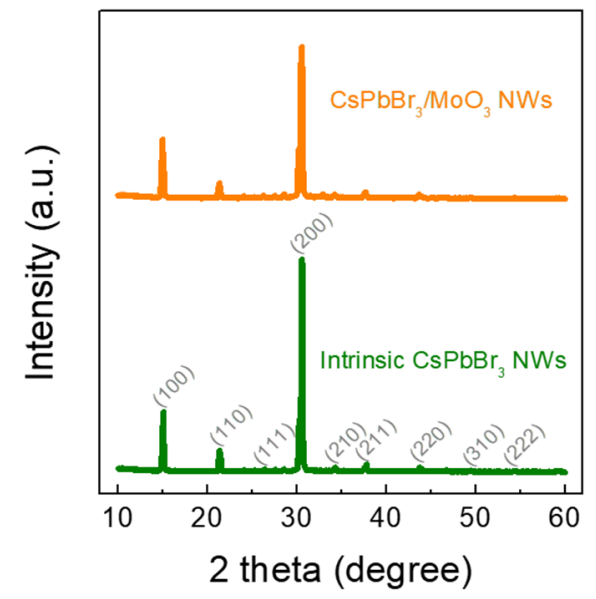

Figure S6. XRD patterns of pristine $\mathrm{CsPbBr} 3 \mathrm{NWs}$ and $\mathrm{CsPbBr} 3 / \mathrm{MoO}_{3}$ core-shell NWs.

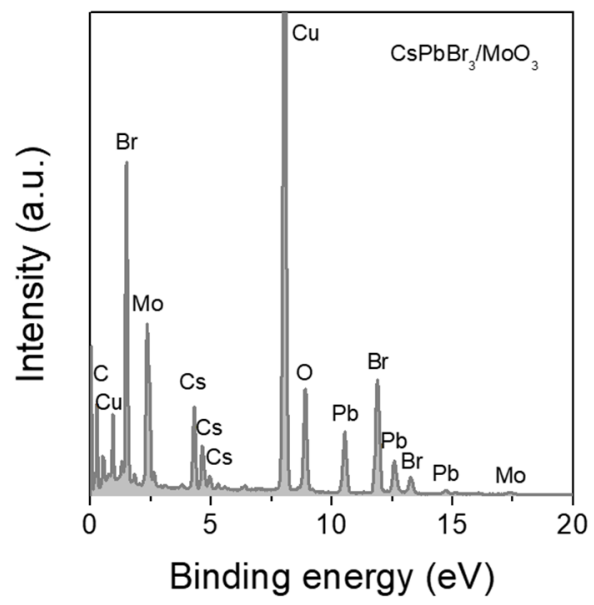

Figure S7. EDS analysis of VLS-grown $\mathrm{CsPbBr} 3 / \mathrm{MoO}_{3}$ core-shell NWs.

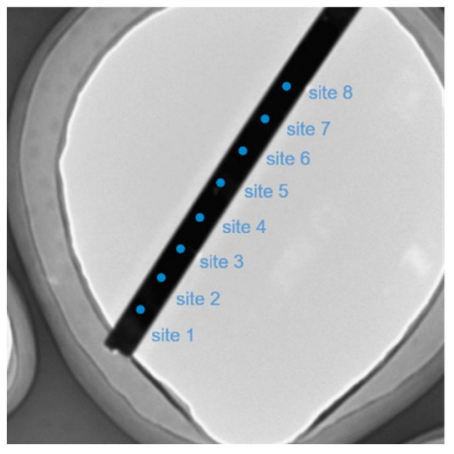

\begin{tabular}{l|lllll}
\hline Spectrum & In stats. & $\mathrm{Br}$ & $\mathrm{Mo}$ & $\mathrm{Cs}$ & $\mathrm{Pb}$ \\
& & & & & \\
Spectrum 1 & Yes & 51.03 & 11.15 & 19.81 & 18.00 \\
Spectrum 2 & Yes & 50.22 & 10.20 & 19.91 & 19.67 \\
Spectrum 3 & Yes & 52.91 & 11.06 & 19.50 & 16.54 \\
Spectrum 4 & Yes & 51.35 & 12.84 & 17.17 & 18.64 \\
Spectrum 5 & Yes & 52.35 & 11.16 & 18.17 & 18.32 \\
Spectrum 6 & Yes & 53.06 & 10.19 & 18.90 & 17.85 \\
Spectrum 7 & Yes & 53.02 & 9.20 & 19.44 & 18.34 \\
Spectrum 8 & Yes & 50.06 & 12.18 & 18.50 & 19.26 \\
& & & & & \\
Mean & & 51.75 & 10.99 & 18.93 & 18.33 \\
Std. deviation & & 1.25 & 1.16 & 0.94 & 0.95 \\
Max. & & 53.06 & 12.84 & 19.91 & 19.67 \\
Min. & & 50.06 & 9.20 & 17.17 & 16.54 \\
\hline All results in atomic\% & & & &
\end{tabular}

Figure S8. EDS analysis of different positions along the length of the $\mathrm{CsPbBr} / \mathrm{MoO}_{3}$ core-shell NW and the corresponding composition quantification. 


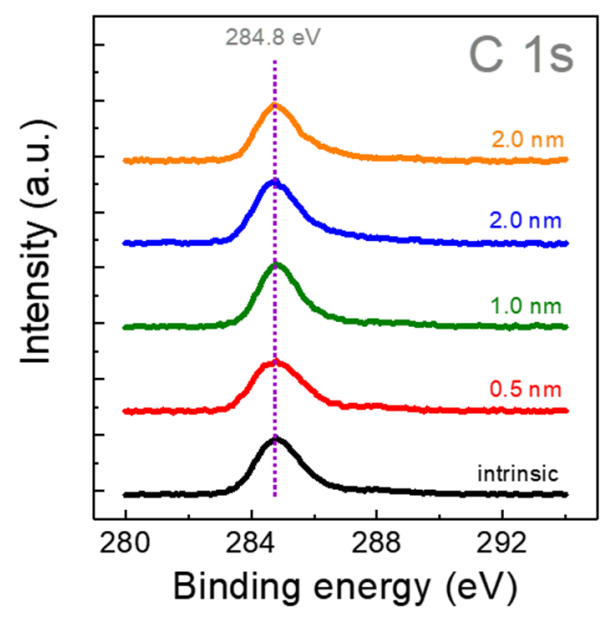

Figure S9. XPS C 1s spectra of $\mathrm{CsPbBr} / \mathrm{MoO}_{3}$ core-shell NWs with different $\mathrm{MoO}_{3}$ thickness.

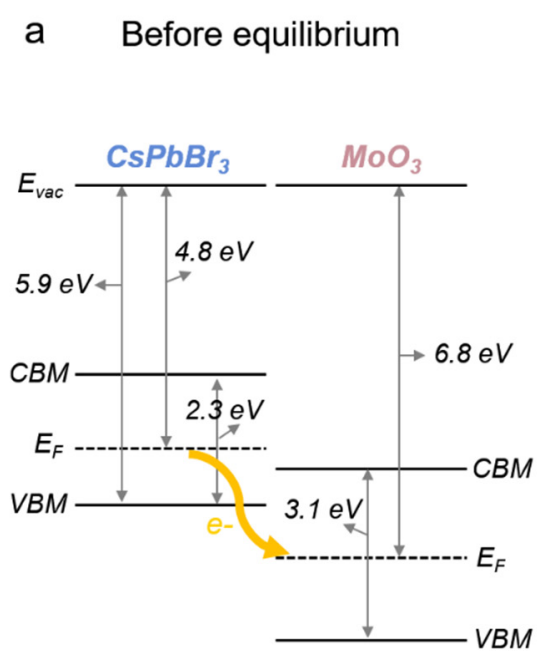

b After equilibrium

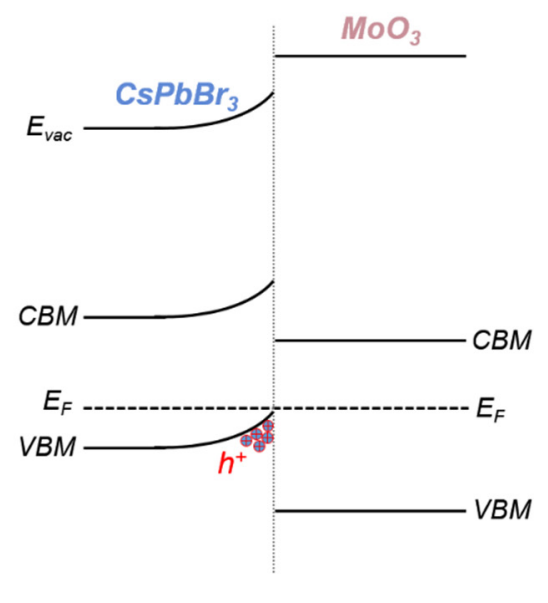

Figure S10. Schematic of the qualitative energy level diagram (a) before and (b) after surface transfer doping using the $\mathrm{MoO}_{3}$ shell layer. When the thickness of $\mathrm{MoO}_{3}$ increases, there are more delocalized holes get accumulated to set up a space charge region at the $\mathrm{CsPbBr}$ side of the $\mathrm{CsPbBr} / \mathrm{MoO}_{3}$ interface, resulting in a more severe upward band bending.

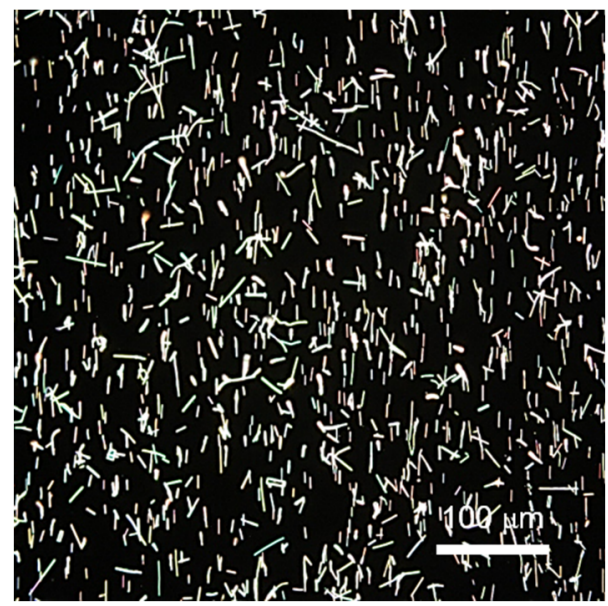

Figure S11. Optical image of $\mathrm{CsPbBr} / \mathrm{MoO}_{3}$ core-shell $\mathrm{NWs}$ laid down on $\mathrm{SiO}_{2} / \mathrm{Si}$ substrates through a dry transfer method. 


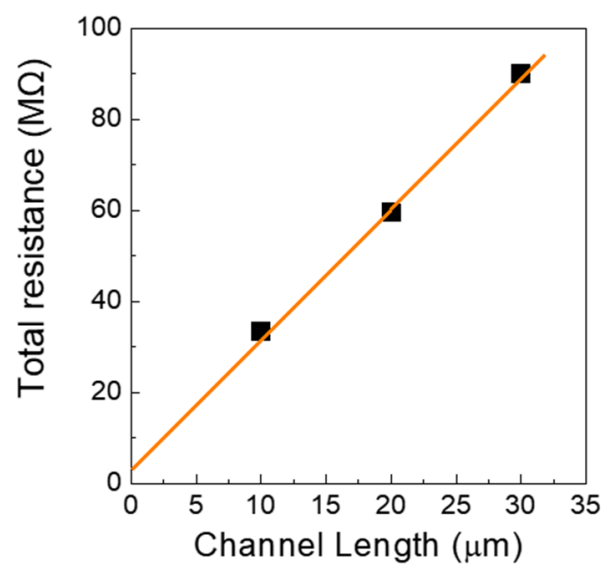

Figure S12. Total resistance as a function of the channel length for the $\mathrm{CsPbBr} 3 / 5-\mathrm{nm} \mathrm{MoO}_{3} \mathrm{NW}$ transistor device.

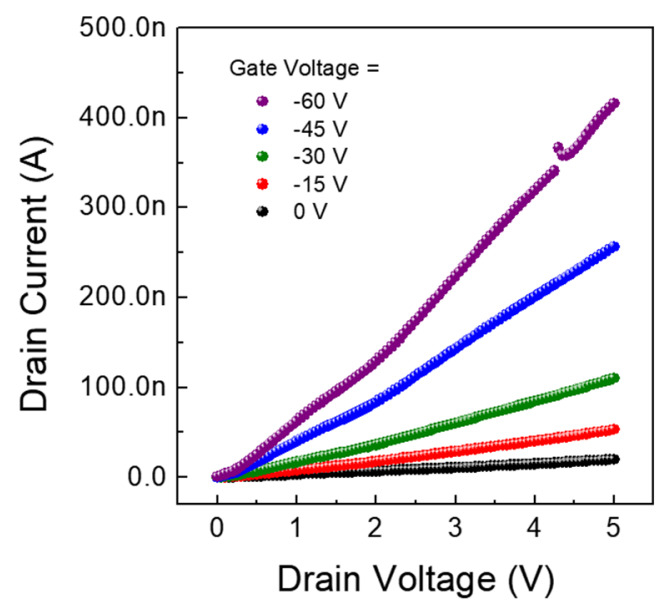

Figure S13. Output characteristics of the single VLS-grown $\mathrm{CsPbBr} 3 / \mathrm{MoO}_{3}$ core-shell NW FET with a $\mathrm{MoO}_{3}$ thickness of $5 \mathrm{~nm}$.

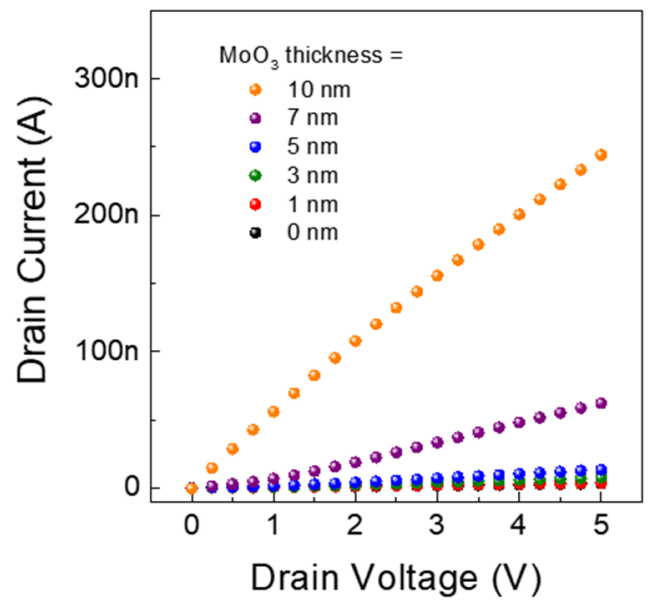

Figure S14. Source-drain IV measurement of $\mathrm{CsPbBr} / \mathrm{MoO}_{3} \mathrm{NW}$ devices with a zero gate bias as a function of $\mathrm{MoO}_{3}$ thickness. 


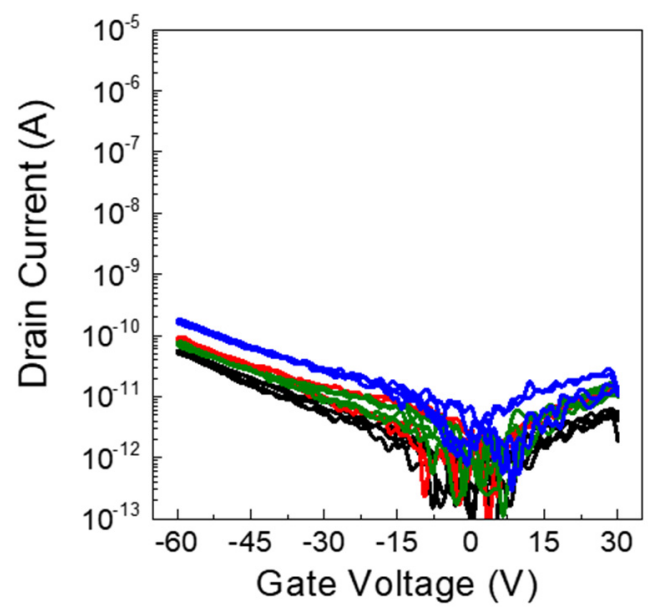

Figure S15. Transfer curves of thin film transistors using 50-nm-thick $\mathrm{MoO}_{3}$ layer as the device channel.

a. cylinder on-plane model

b. parallel plate model
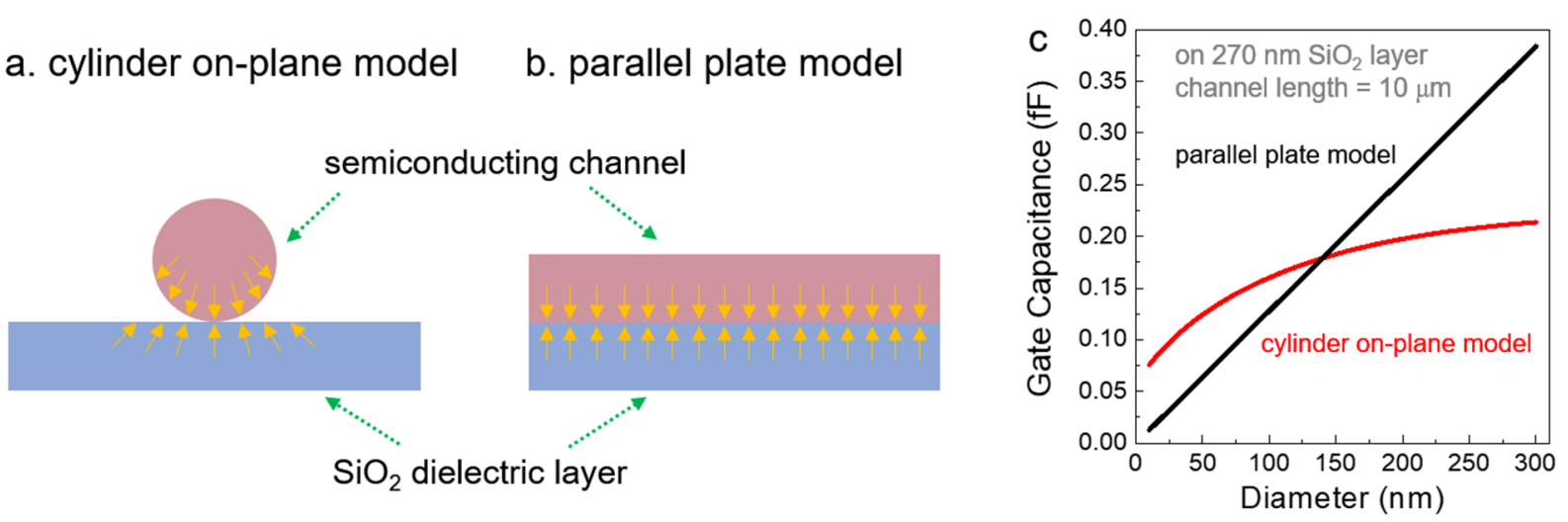

Figure S16. Determination of the gate capacitance of nanowire devices in (a) cylinder on-plane capacitance model and (b) parallel plate capacitance model. (c) The gate capacitance as a function of diameter in the cylinder on-plane capacitance model and the parallel plate capacitance model.

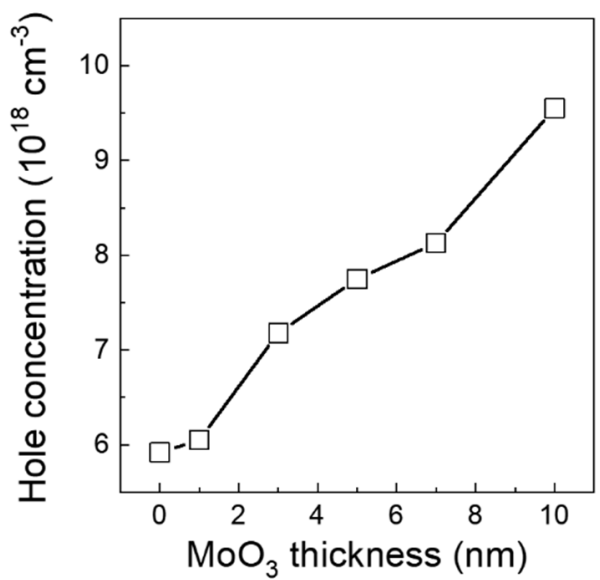

Figure S17. Hole concentration of $\mathrm{CsPbBr} 3 / \mathrm{MoO}_{3}$ core-shell NW FETs with the increasing $\mathrm{MoO}_{3}$ thickness (at $\left.\mathrm{V}_{\mathrm{g}}=-60 \mathrm{~V}\right)$. 

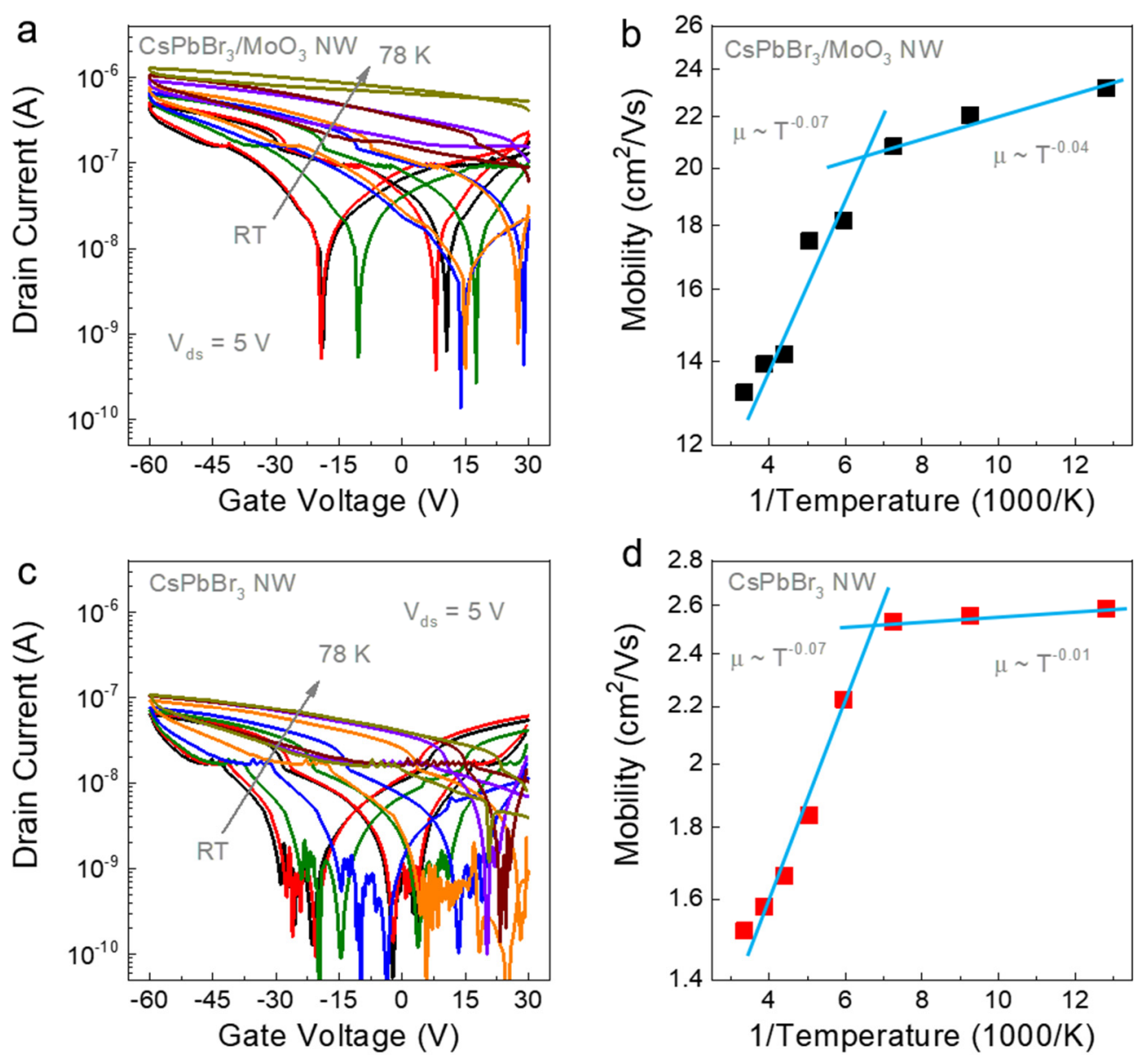

Figure S18. (a) Temperature dependent transfer curves and (b) extracted field-effect hole mobility of the $\mathrm{CsPbBr}_{3} / 5-\mathrm{nm} \mathrm{MoO}_{3} \mathrm{NW}$ transistor. (c) Temperature dependent transfer curves and (d) extracted field-effect mobility of the $\mathrm{CsPBBr}_{3} \mathrm{NW}$ transistor. The devices are measured with a temperature range between $298 \mathrm{~K}$ and $78 \mathrm{~K}$. 

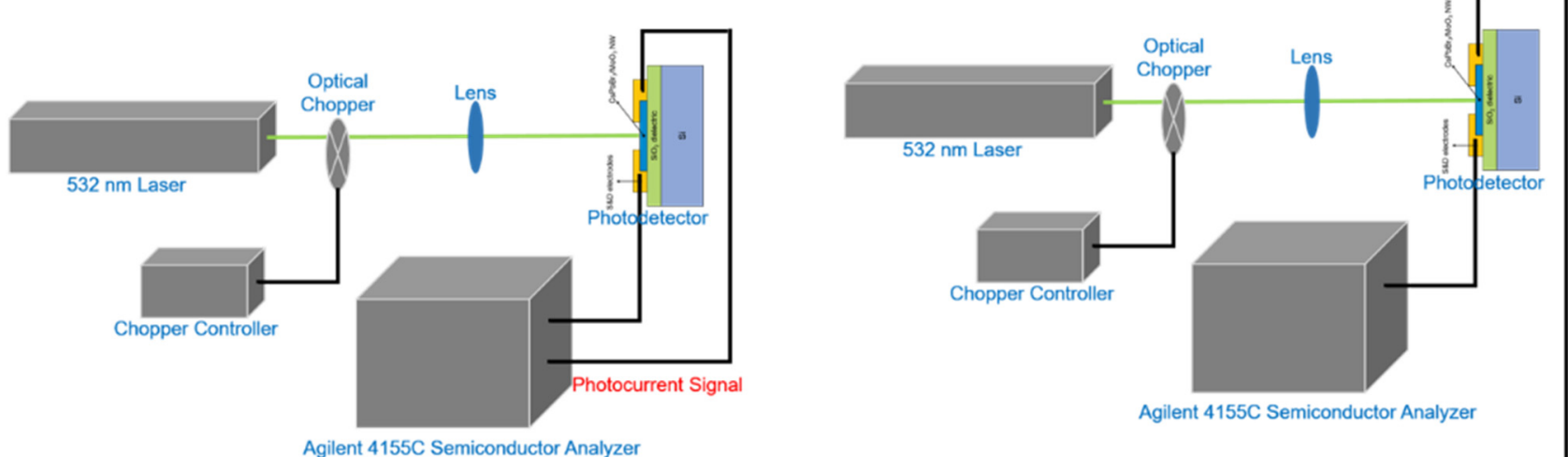

Agilent $4155 \mathrm{C}$ Semiconductor Analyzer

Agilent 4155C Semiconductor Analyzer

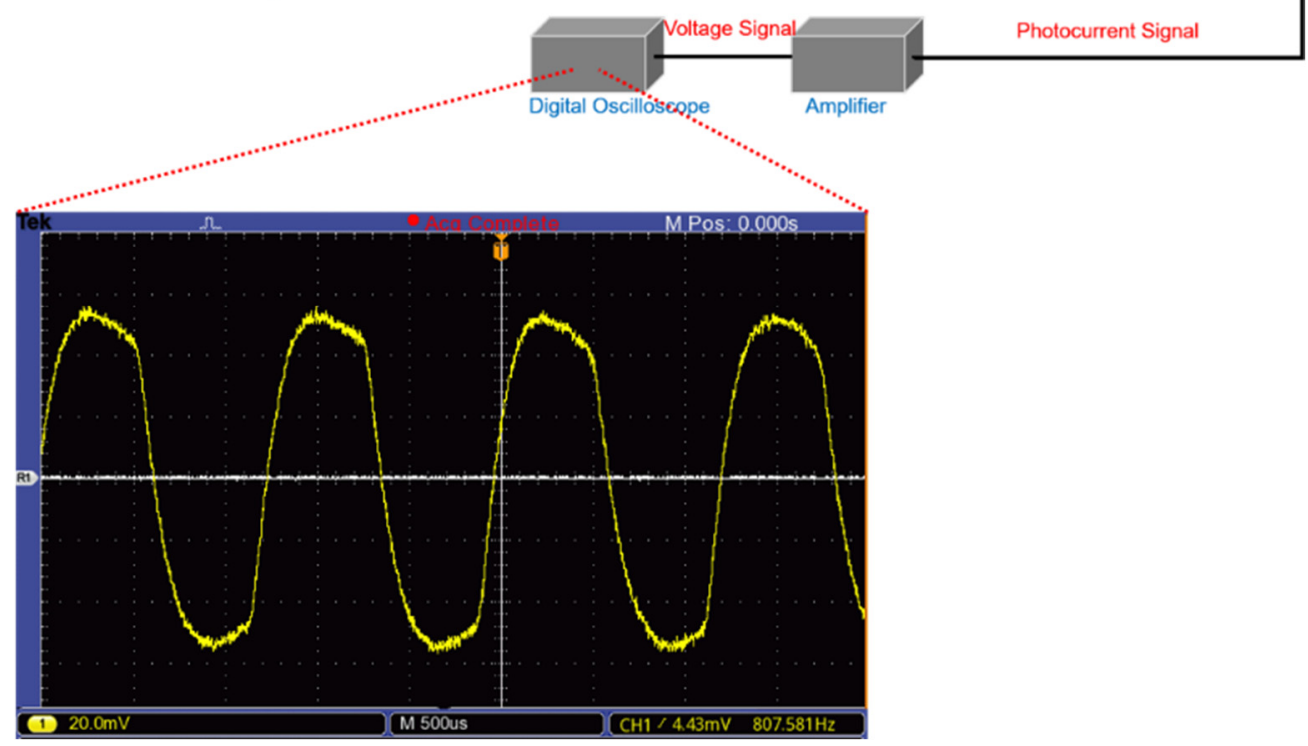

Figure S19. Experimental setup of the photodetector measurement under (a) low chopping frequency and (b) high chopping frequency. 

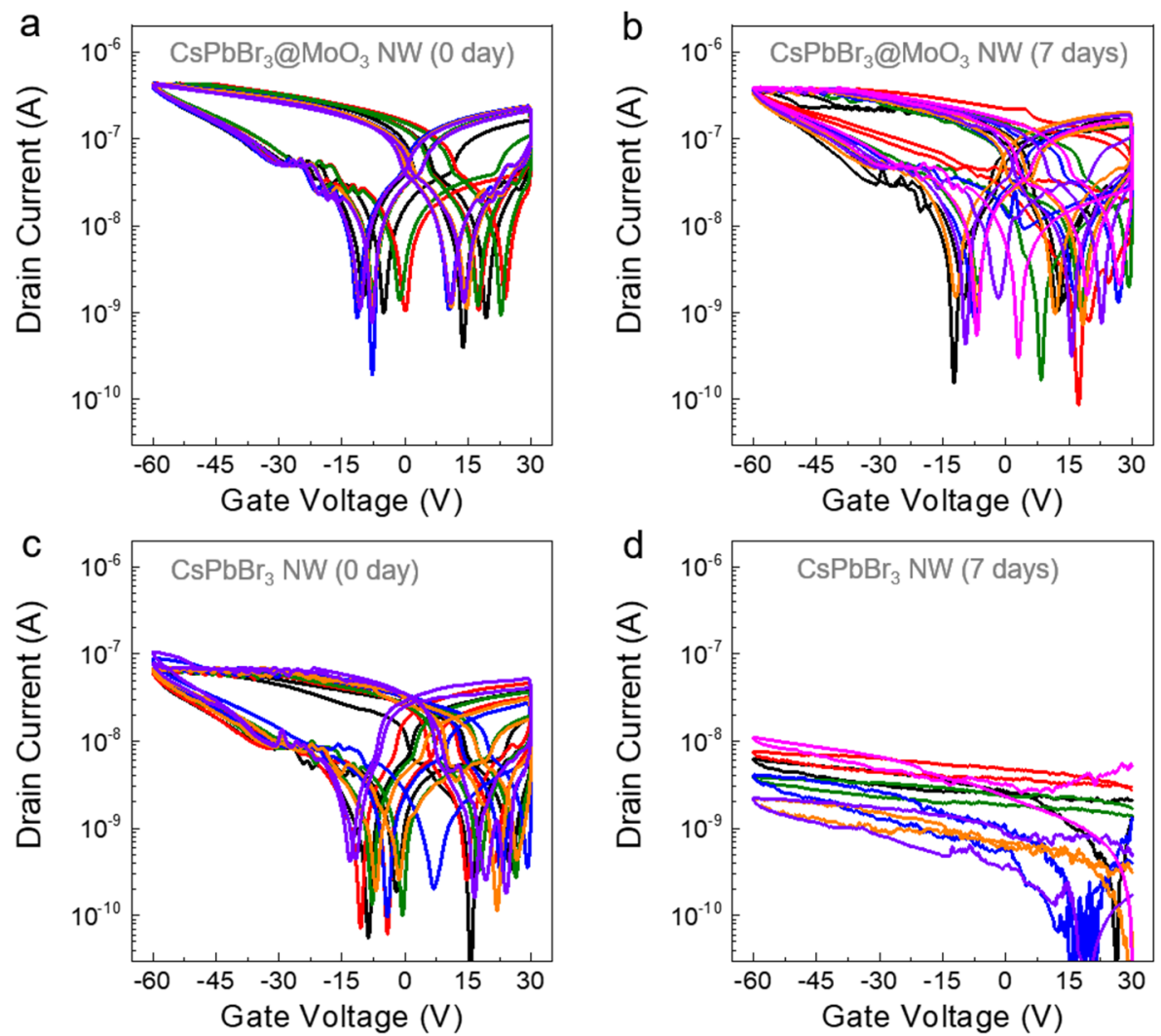

Figure S20. Transfer curves of the (a and b) $\mathrm{CsPbBr} 3 / \mathrm{MoO}_{3} \mathrm{NW}$ device and (c and d) pristine $\mathrm{CsPbBr}_{3} \mathrm{NW}$ device with different duration of storage.

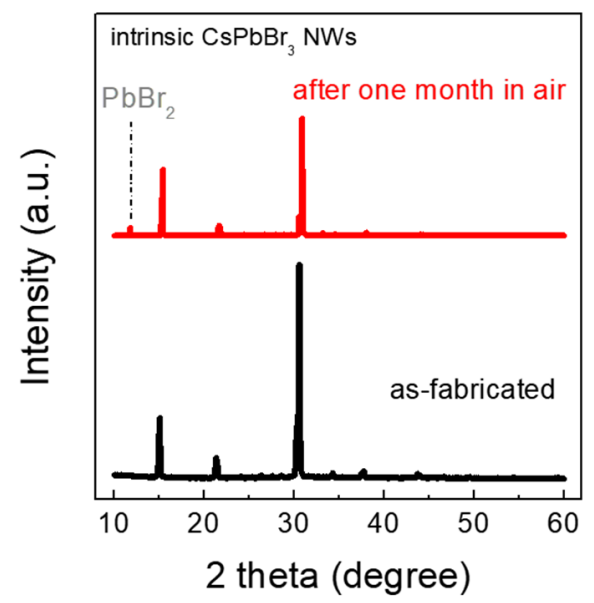

Figure S21. XRD patterns of pristine $\mathrm{CsPbBr}_{3} \mathrm{NWs}$ measured after material fabrication and storage in ambient conditions for a period of 30 days. 


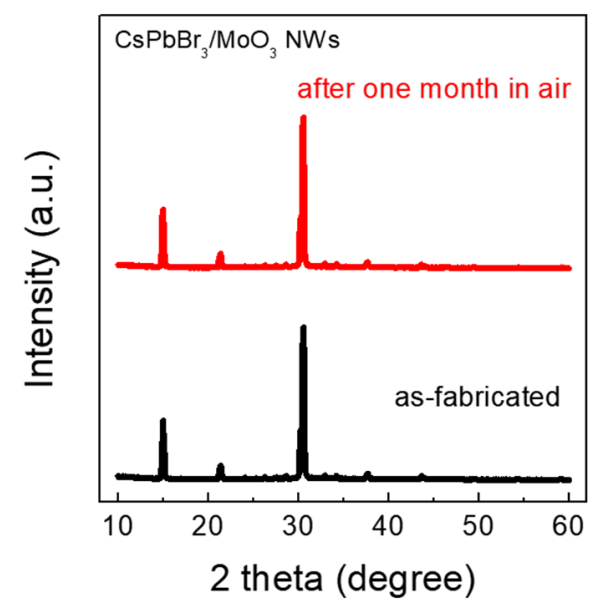

Figure S22. XRD patterns of $\mathrm{CsPbBr} 3 / \mathrm{MoO}_{3}$ core-shell NWs measured after material fabrication and storage in ambient conditions for a period of 30 days.

Table S1. Energy level values of different transition metal oxides that may be used as electronwithdrawing surface dopants.

\begin{tabular}{cccc}
\hline \hline Material & $\begin{array}{c}\text { Ionization Energy } \\
(\mathrm{IE})\end{array}$ & $\begin{array}{c}\text { Work Function } \\
(\mathrm{WF})\end{array}$ & $\begin{array}{c}\text { Electron Affinity } \\
(\mathrm{EA})\end{array}$ \\
\hline $\mathrm{MoO}_{3}$ & $9.6 \mathrm{eV}$ & $6.8 \mathrm{eV}$ & $6.4 \mathrm{eV}$ \\
$\mathrm{WO}_{3}$ & $9.8 \mathrm{eV}$ & $6.7 \mathrm{eV}$ & $6.5 \mathrm{eV}$ \\
$\mathrm{CrO}_{3}$ & $9.0 \mathrm{eV}$ & $6.8 \mathrm{eV}$ & $6.7 \mathrm{eV}$ \\
$\mathrm{V}_{2} \mathrm{O}_{5}$ & $9.5 \mathrm{eV}$ & $7.0 \mathrm{eV}$ & $6.7 \mathrm{eV}$ \\
$\mathrm{ReO}_{3}$ & - & $6.8 \mathrm{eV}$ & - \\
\hline \hline
\end{tabular}


Table S2. Performance summary of photodetectors based on low-dimensional halide perovskites.

\begin{tabular}{|c|c|c|c|c|c|c|c|c|}
\hline Year & Material & Channel & Method & $\begin{array}{c}\text { Responsivity } \\
{[\mathrm{A} / \mathrm{W}]}\end{array}$ & $\begin{array}{l}\text { On/off } \\
\text { ratio }\end{array}$ & $\begin{array}{c}\text { Detectivity } \\
\text { [Jones] }\end{array}$ & $\begin{array}{c}\text { Rise/decay } \\
\text { time [ms] }\end{array}$ & Ref. \\
\hline 2014 & $\mathrm{MAPbI}_{3}$ & NWs & Solution & 0.005 & $10^{2}$ & - & $0.35 / 0.25$ & 1 \\
\hline 2015 & $\mathrm{MAPbI}_{3}$ & NWs & Solution & 0.1 & 300 & $1.02 \times 10^{12}$ & $0.3 / 0.4$ & 2 \\
\hline 2015 & $\mathrm{MAPbI}_{3}$ & NWs & Solution & 1.32 & 20 & $2.5 \times 10^{12}$ & $0.2 / 0.3$ & 3 \\
\hline 2016 & $\mathrm{CsPbBr}_{3}$ & MWs & Vapor & 0.001 & $>10^{3}$ & - & $0.1 / 0.1$ & 4 \\
\hline 2016 & $\mathrm{CsPbBr}_{3}$ & NPs & Solution & 0.64 & $>10^{3}$ & - & $0.019 / 0.024$ & 5 \\
\hline 2016 & $\mathrm{CsPbI}_{3}$ & NCs & Solution & - & $>10^{5}$ & - & $24 / 29$ & 6 \\
\hline 2016 & $\mathrm{CsPbBr}_{3}$ & NCs & Solution & 0.18 & $>10^{3}$ & - & $1.8 / 1$ & 7 \\
\hline 2016 & $\mathrm{CsPb}(\mathrm{Br} / \mathrm{I})_{3}$ & NWs & Solution & - & $>10^{3}$ & - & $680 / 660$ & 8 \\
\hline 2016 & $\mathrm{CsPbBr}_{3}$ & NPs & Solution & - & $10^{2}$ & - & $17.8 / 14.7$ & 9 \\
\hline 2016 & $\mathrm{MAPbI}_{3}$ & NWs & Vapor & - & - & $10^{10}$ & $0.205 / 0.138$ & 10 \\
\hline 2016 & $\mathrm{MASnI}_{3}$ & NWs & Vapor & 0.47 & - & $8.80 \times 10^{10}$ & $1500 / 400$ & 11 \\
\hline 2016 & $\mathrm{MAPbI}_{3}$ & NPs & Combined & 22 & $10^{2}$ & - & $<20 /<40$ & 12 \\
\hline 2016 & $\mathrm{MAPbI}_{3}$ & NPs & Vapor & 0.025 & $<10$ & - & $150 / 150$ & 13 \\
\hline 2016 & $\mathrm{MAPbI}_{3}$ & MWs & Solution & 13.5 & $10^{2}$ & $5.25 \times 10^{12}$ & $0.08 / 0.24$ & 14 \\
\hline 2016 & $\mathrm{MAPbI}_{3}$ & NWs & Solution & 4.95 & 4000 & $2 \times 10^{13}$ & $<0.1 /<0.1$ & 15 \\
\hline 2016 & $\mathrm{MAPbI}_{3}$ & NWs & Solution & - & 13 & - & $120 / 210$ & 16 \\
\hline 2017 & $\mathrm{CsPbBr}_{3}$ & NPs & Solution & 34 & 5000 & $7.5 \times 10^{12}$ & $0.6 / 0.9$ & 17 \\
\hline 2017 & $\mathrm{CsPbBr}_{3}$ & NPs & Solution & 31.1 & $10^{3}$ & - & $0.016 / 0.38$ & 18 \\
\hline 2017 & $\mathrm{CsPbBr}_{3}$ & NWs & Solution & 1377 & $10^{3}$ & - & $0.022 / 0.023$ & 19 \\
\hline 2017 & $\mathrm{CsPbI}_{3}$ & NWs & Vapor & 0.0067 & - & $1.57 \times 10^{8}$ & $292 / 234$ & 20 \\
\hline 2018 & $\mathrm{CsPbBr}_{3}$ & NWs & Vapor & - & $10^{3}$ & - & $0.01 / 0.005$ & 21 \\
\hline 2018 & $\mathrm{MAPbI}_{3}$ & $\begin{array}{l}\text { Single- } \\
\text { NW }\end{array}$ & Solution & 410 & 740 & $9.1 \times 10^{12}$ & $0.22 / 0.79$ & 22 \\
\hline 2019 & $\mathrm{CsPbCl}_{3}$ & $\begin{array}{l}\text { Single- } \\
\text { NW }\end{array}$ & Vapor & 1183 & $>10^{4}$ & $6.6 \times 10^{12}$ & $<50 /<50$ & 23 \\
\hline 2020 & $\begin{array}{l}\mathrm{CsPbBr}_{3} \\
@ \mathrm{MoO}_{3}\end{array}$ & $\begin{array}{l}\text { Single- } \\
\text { NW }\end{array}$ & Vapor & 2360 & $>10^{4}$ & $2.1 \times 10^{12}$ & $0.23 / 0.28$ & $\begin{array}{l}\text { This } \\
\text { work }\end{array}$ \\
\hline
\end{tabular}

NWs = Nanowires; NPs = Nanoplatelets; NCs = Nanocrystals; '-' = Not Given. 


\section{References}

1. Horvath, E.; Spina, M.; Szekrenyes, Z.; Kamaras, K.; Gaal, R.; Gachet, D.; Forro, L., Nanowires of Methylammonium Lead Iodide $\left(\mathrm{CH}_{3} \mathrm{NH}_{3} \mathrm{PbI}_{3}\right)$ Prepared by Low Temperature SolutionMediated Crystallization. Nano Lett. 2014, 14, 6761-6766.

2. Deng, H.; Yang, X.; Dong, D.; Li, B.; Yang, D.; Yuan, S.; Qiao, K.; Cheng, Y. B.; Tang, J.; Song, H., Flexible and Semitransparent Organolead Triiodide Perovskite Network Photodetector Arrays with High Stability. Nano Lett. 2015, 15, 7963-7969.

3. Deng, H.; Dong, D.; Qiao, K.; Bu, L.; Li, B.; Yang, D.; Wang, H. E.; Cheng, Y.; Zhao, Z.; Tang, J.; Song, H., Growth, Patterning and Alignment of Organolead Iodide Perovskite Nanowires for Optoelectronic Devices. Nanoscale 2015, 7, 4163-4170.

4. Chen, J.; Fu, Y.; Samad, L.; Dang, L.; Zhao, Y.; Shen, S.; Guo, L.; Jin, S., Vapor-Phase Epitaxial Growth of Aligned Nanowire Networks of Cesium Lead Halide Perovskites $\left(\mathrm{CsPbX}_{3}, \mathrm{X}=\right.$ Cl, Br, I). Nano Lett. 2017, 17, 460-466.

5. Song, J.; Xu, L.; Li, J.; Xue, J.; Dong, Y.; Li, X.; Zeng, H., Monolayer and Few-Layer AllInorganic Perovskites as a New Family of Two-Dimensional Semiconductors for Printable Optoelectronic Devices. Adv. Mater. 2016, 28, 4861-4869.

6. Ramasamy, P.; Lim, D. H.; Kim, B.; Lee, S. H.; Lee, M. S.; Lee, J. S., All-Inorganic Cesium Lead Halide Perovskite Nanocrystals for Photodetector Applications. Chem. Commun. 2016, 52, 2067-2070.

7. Li, X.; Yu, D.; Cao, F.; Gu, Y.; Wei, Y.; Wu, Y.; Song, J.; Zeng, H., Healing All-Inorganic Perovskite Films via Recyclable Dissolution-Recyrstallization for Compact and Smooth Carrier Channels of Optoelectronic Devices with High Stability. Adv. Funct. Mater. 2016, 26, 5903-5912.

8. Tang, X.; Zu, Z.; Shao, H.; Hu, W.; Zhou, M.; Deng, M.; Chen, W.; Zang, Z.; Zhu, T.; Xue, J., All-Inorganic Perovskite $\mathrm{CsPb}(\mathrm{Br} / \mathrm{I})_{3}$ Nanorods for Optoelectronic Application. Nanoscale 2016, 8, 15158-15161.

9. Lv, L.; Xu, Y.; Fang, H.; Luo, W.; Xu, F.; Liu, L.; Wang, B.; Zhang, X.; Yang, D.; Hu, W.; Dong, A., Generalized Colloidal Synthesis of High-Quality, Two-Dimensional Cesium Lead Halide Perovskite Nanosheets and Their Applications in Photodetectors. Nanoscale 2016, 8, 13589-13596.

10. Gu, L.; Tavakoli, M. M.; Zhang, D.; Zhang, Q.; Waleed, A.; Xiao, Y.; Tsui, K. H.; Lin, Y.; Liao, L.; Wang, J.; Fan, Z., 3D Arrays of 1024-Pixel Image Sensors Based on Lead Halide Perovskite Nanowires. Adv. Mater. 2016, 28, 9713-9721.

11. Waleed, A.; Tavakoli, M. M.; Gu, L.; Wang, Z.; Zhang, D.; Manikandan, A.; Zhang, Q.; Zhang, 
R.; Chueh, Y. L.; Fan, Z., Lead-Free Perovskite Nanowire Array Photodetectors with Drastically Improved Stability in Nanoengineering Templates. Nano Lett. 2017, 17, 523-530.

12. Liu, J.; Xue, Y.; Wang, Z.; Xu, Z. Q.; Zheng, C.; Weber, B.; Song, J.; Wang, Y.; Lu, Y.; Zhang, Y.; Bao, Q., Two-Dimensional $\mathrm{CH}_{3} \mathrm{NH}_{3} \mathrm{PbI}_{3}$ Perovskite: Synthesis and Optoelectronic Application. ACS Nano 2016, 10, 3536-3542.

13. Niu, L.; Zeng, Q.; Shi, J.; Cong, C.; Wu, C.; Liu, F.; Zhou, J.; Fu, W.; Fu, Q.; Jin, C.; Yu, T.; Liu, X.; Liu, Z., Controlled Growth and Reliable Thickness-Dependent Properties of OrganicInorganic Perovskite Platelet Crystal. Adv. Funct. Mater. 2016, 26, 5263-5270.

14. Deng, W.; Zhang, X.; Huang, L.; Xu, X.; Wang, L.; Wang, J.; Shang, Q.; Lee, S. T.; Jie, J., Aligned Single-Crystalline Perovskite Microwire Arrays for High-Performance Flexible Image Sensors with Long-Term Stability. Adv. Mater. 2016, 28, 2201-2208.

15. Gao, L.; Zeng, K.; Guo, J.; Ge, C.; Du, J.; Zhao, Y.; Chen, C.; Deng, H.; He, Y.; Song, H.; Niu, G.; Tang, J., Passivated Single-Crystalline $\mathrm{CH}_{3} \mathrm{NH}_{3} \mathrm{PbI}_{3}$ Nanowire Photodetector with High Detectivity and Polarization Sensitivity. Nano Lett. 2016, 16, 7446-7454.

16. Zhu, P.; Gu, S.; Shen, X.; Xu, N.; Tan, Y.; Zhuang, S.; Deng, Y.; Lu, Z.; Wang, Z.; Zhu, J., Direct Conversion of Perovskite Thin Films into Nanowires with Kinetic Control for Flexible Optoelectronic Devices. Nano Lett. 2016, 16, 871-876.

17. Liu, X.; Yu, D.; Cao, F.; Li, X.; Ji, J.; Chen, J.; Song, X.; Zeng, H., Low-Voltage Photodetectors with High Responsivity Based on Solution-Processed Micrometer-Scale AllInorganic Perovskite Nanoplatelets. Small 2017, 13, 1700364.

18. Li, X.; Yu, D.; Chen, J.; Wang, Y.; Cao, F.; Wei, Y.; Wu, Y.; Wang, L.; Zhu, Y.; Sun, Z.; Ji, J.; Shen, Y.; Sun, H.; Zeng, H., Constructing Fast Carrier Tracks into Flexible Perovskite Photodetectors to Greatly Improve Responsivity. ACS Nano 2017, 11, 2015-2023.

19. Feng, J.; Yan, X.; Liu, Y.; Gao, H.; Wu, Y.; Su, B.; Jiang, L., Crystallographically Aligned Perovskite Structures for High-Performance Polarization-Sensitive Photodetectors. Adv. Mater. 2017, $29,1605993$.

20. Waleed, A.; Tavakoli, M. M.; Gu, L.; Hussain, S.; Zhang, D.; Poddar, S.; Wang, Z.; Zhang, R.; Fan, Z., All Inorganic Cesium Lead Iodide Perovskite Nanowires with Stabilized Cubic Phase at Room Temperature and Nanowire Array-Based Photodetectors. Nano Lett. 2017, 17, 4951-4957.

21. Oksenberg, E.; Sanders, E.; Popovitz-Biro, R.; Houben, L.; Joselevich, E., Surface-Guided $\mathrm{CsPbr}_{3}$ Perovskite Nanowires on Flat and Faceted Sapphire with Size-Dependent Photoluminescence and Fast Photoconductive Response. Nano Lett. 2018, 18, 424-433. 
22. Zhou, Q.; Park, J. G.; Nie, R.; Thokchom, A. K.; Ha, D.; Pan, J.; Seok, S. I.; Kim, T., Nanochannel-Assisted Perovskite Nanowires: From Growth Mechanisms to Photodetector Applications. ACS Nano 2018, 12, 8406-8414.

23. Meng, Y.; Lan, C.; Li, F.; Yip, S.; Wei, R.; Kang, X.; Bu, X.; Dong, R.; Zhang, H.; Ho, J. C., Direct Vapor-Liquid-Solid Synthesis of All-Inorganic Perovskite Nanowires for High-Performance Electronics and Optoelectronics. ACS Nano 2019, 13, 6060-6070. 